\title{
Parallel Palm print Identification on GPU
}

\author{
N.V.Rohit ${ }^{1}$, Mayur Vikas Patil ${ }^{2}$, Yogesh Patil ${ }^{3}$, Kiran Muske ${ }^{4}$, Prof.Santwana S. Gudadhe ${ }^{5}$ Prof. Dr.J.S.Umale \\ Department of Computer Engineering, Pimpri Chinchwad College of Engineering, Pune, India ${ }^{1,2,3,4,5,6}$
}

Abstract: Biometric technologies are becoming inevitable in highly secure identification and personal verification solutions. With an increase in transaction frauds, security breaches the need for highly secure identification and personal verification technologies is becoming need of the hour. The major advantage of biometric system over traditional methods is that they are typically unique for each person and cannot be forged easily. Biometric use of palm prints uses ridge patterns to identify an individual. The Palm print identification system is most promising to identify an individual based on statistical properties of palm print image. Its rich features like principal lines, minutiae points, wrinkles, ridges and singular points makes it favourable to be used as one of the best biometric identification. This paper proposed the palm print identification system on GPU.

Keywords: Palm print identification, Biometrics, fractional coefficient, Graphical Processing Unit (GPU) , Discrete Cosine transform (DCT) , Genuine Acceptance Ratio (GAR)

\section{INTRODUCTION}

Biometrics is used for authentication and identification. It is universally accepted as well as the use of the system for verification has been accepted by masses. Verifying identity based on physical characteristics or behavioral patterns is known as biometric [9]. Biometric use for identification and verification involve iris, DNA, finger print, palm print, face detection etc. Each of these has its advantages and disadvantages as composed with all techniques. Palm print has rich features and makes it favorable to be used as one of the best biometric identification.

Palm print identification uses the person's palm as a biometric for identifying or verifying person's identity. Palm print patterns are a very reliable biometric and require minimum cooperation from the user for extraction due to our mobility of our hand towards any device for scanning. Palm print is distinctive, easily captured by low resolution devices; we may need 150 dpi or less for commercial purposes and nearly $400 \mathrm{dpi}$ or less for scientific use. Therefore it is suitable for everyone and it does not require any other personal information of the user. In this paper, basic palm print identification system and various approaches are discussed. This paper proposed the palm print identification system using the concept of fractional coefficient [1] on GPU.

\section{BASIC PALM PRINT IDENTIFICATION}

In the basic palm print scanning, we can use various devices which even work with low resolution palm images. It can also involve touch-less as well as scanner which needs physical contact of palm.

Palm-print is the region between wrist and fingers and has principle lines, wrinkles, ridges, minutiae points, singular points and texture pattern which can be considered as biometric characteristics. Palm print consists of principal lines, wrinkles and ridges. The principal lines are the main line present in most of the people palm print. Heart line, headline and lifeline are the three types of principle lines. Ridges and wrinkles are the coarse and fine line of the palm print. Palm print recognition uses the person's palm as a biometric for identifying or verifying who the person is? Palm print has three modes: Enrollment, identification and verification.

Palm print identification system consists of image acquisition in which image is capture with the help of device. Preprocessing sets up coordinate system to align palm print images and to segment a part of palm print image for feature extraction. It obtains effective features. The matcher compares the extracted features with the features stored in the database. Fig. 1 shows the basic palm print identification system.

Some palm identification systems operate by scanning the entire palm, whereas others require the palms to be segmented into smaller areas to optimize performance. Reliability of palm print identification can be improved by searching the smaller data sets. Palm print recognition is shown in the Fig. 1 and explained below [20].

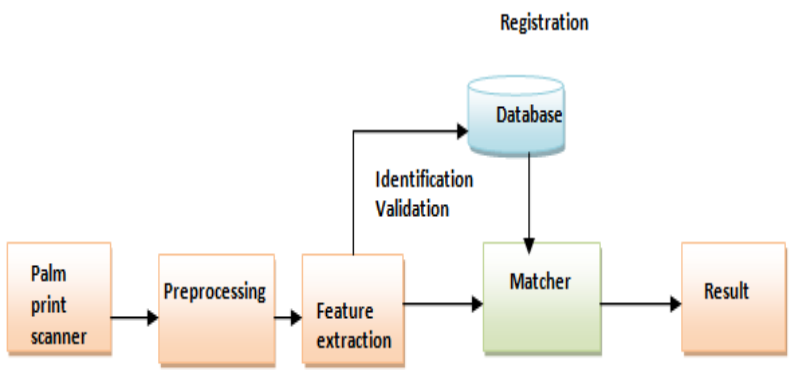

Figure 1. Palm Print Identification System

\section{A. Image Acquisition}

Palm print Scanner- The palms print scanner collects palm print images. There are four type of scanner used by the researchers. CCD based palm print scanner, digital camera, digital scanner and video camera for collecting the palm print image.

\section{I) Preprocessing}

Preprocessing- in preprocessing techniques different palm print images are aligned and central part of image is extracted for feature extraction.

Preprocessing involve five common steps- 
1. Binarizing the palm print image.

2. Extracting the contour lines of hand or finger.

3. Detecting the key point.

4. Establishing a coordination system.

5. Extracting central part.

\section{II) Feature Extraction}

Feature extraction is followed by pre-processing [1]. In feature extraction phase features of palm are extracted like principal lines, orientation field, minutiae, density map, texture, singular points etc.

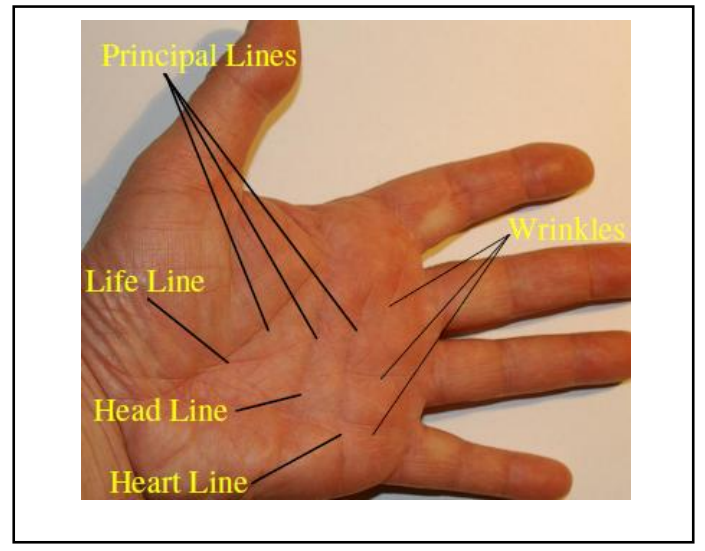

Figure 2. Palm's feature points

Matching is next to the feature extraction phase. Feature matching determines the degree of similarity of recognition template with master template. Different approaches are used for matching. Input provided by individual is matched with templates present in database

\section{B) Matcher}

After the feature extraction, the feature vectors are matched with the feature vectors stored in database. Various recognition algorithms are used for matching according to the feasibility as well as considering the accuracy of matching the palm image with the existing image in the database.

\section{C) Database}

Standard palm print databases available are listed below:

1) IIT Delhi Touch less Palm print Database (Version 1.0),[7] http://web.iitd.ac.in/ ajaykr/Database_Palm.htm

2) CASIA palm print data base [8] http://biometrics.idealtest.org/dbDetailForUsr.do?id=5

\section{B) VARIOUS APPROACHES}

Many researchers are paying attention on feature extraction and matching method. Generally all techniques are classifiede into two type, spatial domain and frequency domain. Both the domain has got its own importance in terms of performance as well as feasibility.
Fig. 3 show the tree structure of various techniques under different domains.

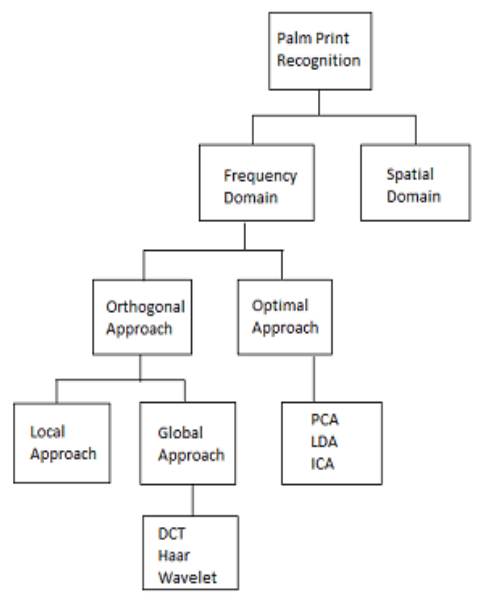

Figure 3. Various approaches

\section{I) SPATIAL APPROACHES:}

In Spatial approach also known as line based approach. In this approach existing edge detectors are used to extract the palm lines are matched directly or represent in other format for matching.

$\mathrm{Wu}$ et al. describes the Sobel mask to calculate the magnitude of palm lines [11]. The histogram is formed by projecting the calculated magnitude along $\mathrm{X}$ and $\mathrm{Y}$ directions. Author uses two masks to calculate the first order derivative and second order derivative of palm print images. The first order derivative of zero crossing is used to identify the edge point and the direction of the edge. The magnitude of the lines is identified by the Second order derivative. The weighted some of the local directional magnitude is considered as a feature vector. Here Euclidian distance is used for matching.

Kung et al. uses the feature vector which is 18 by 16 dimensional feature vectors based on low resolution edge maps [12]. The feature vector is passed into decision based neural network.

Huang et al. Suggest the two level modified finite random transform and dynamic threshold to extract principal lines [13]. Two binary edge maps are compared based on a matching scheme. This matching scheme is known as pixel-to-area comparison.

\section{A) Frequency based approaches}

In frequency domain approach, it includes orthogonal approach and optimal approach.

\section{1) OPTIMAL APPROACHES:}

Optimal approach is also known as subspace approach. It involves involve principal component analysis (PCA), Linear discriminant analysis (LDA) and independent component analysis (ICA). The subspace coefficients are considered as features. Many researchers also use 
wavelets, discrete cosine transform methods and PCA, LDA, ICA.

Dale et al. proposed discrete cosine transform (DCT) [3],[4] based feature vector for palm print representation and matching compared with wavelet and DFT transform [16].

The Laadjel et al. approach combines fisher's linear discriminant (FLD) and Gabor Wavelet responses. This method involves convolving a palm print image with a series of Gabor wavelets at different scales and rotations before extracting features from the Gabor filtered image [17].

\section{2) Orthogonal approaches:}

Orthogonal approach is also known as statistical approach. This approach is divided into two types: Local approach and global approach.

\section{a) Local Approach:-}

The local approaches transform images into another domain and divide the transform into several regions such as mean and variance of each small region.

Yong et al. method for feature extraction divides the palm print image into a set of $\mathrm{n}$ small regions and then calculates the mean and standard deviation of sub regions. Mostly for matching purpose Euclidian square is used [19].

\section{b) Global Approach:-}

Then global statistical features like moments, center of gravity and density are calculated directly from the whole transformed image.

Dr. H.B Kekre et al. [2] presented that performance comparison of palm print recognition techniques based on fractional coefficients of transformed palm print image using six different transforms like Cosine, Sine, Walsh, Slant, Haar. [2] In transform domain, energy of image gets accumulated towards low frequency region; those characteristic of image transforms was exploited here to reduce the feature vector size of palm print images by neglecting the low frequency coefficients in transformed palm print images. In their method, they described about six image transforms and 13 ways of taking fractional coefficients which result into a total of 78 palm print identification methods. The experimental results in Sine, Walsh, Haar, Cosine transform have shown significant performance improvement in palm print identification using fractional coefficients of transformed images. In all Cosines transform at fractional coefficients of $0.78 \%$ gives best performance as indicated by higher GAR value.

In global approach whole transformed image is considered and feature vectors are computed of this whole transformed image.

The Table I summarize the approaches, the methods used in these approaches and the features considered in respective approaches.
TABLE I

COMPARISON OF VARIOUS APPROACHES

\begin{tabular}{|l|c|c|}
\hline \multirow{2}{*}{ Approach } & Method & Feature \\
\hline \multirow{2}{*}{ OPTIMAL } & $\begin{array}{c}\text { Sobel edge } \\
\text { detector, } \\
\text { Prewitt, Canny }\end{array}$ & Line feature \\
\cline { 2 - 3 } & PCA & Eigen Palm \\
\cline { 2 - 3 } & ICA & Texture feature \\
\hline ORTHOGONAL & $\begin{array}{c}\text { Transform } \\
\text { DCT, Haar }\end{array}$ & $\begin{array}{c}\text { Energy feature } \\
\text { vector }\end{array}$ \\
\hline
\end{tabular}

\section{Proposed System}

\section{A. Proposed Architecture}

In palm print identification, Palm image must be identified to increase the accuracy and speed up the identification process. In proposed identification system, first step is to resize the palm image to standard size, extract the edge and apply the filter to improve the quality and remove the noise.

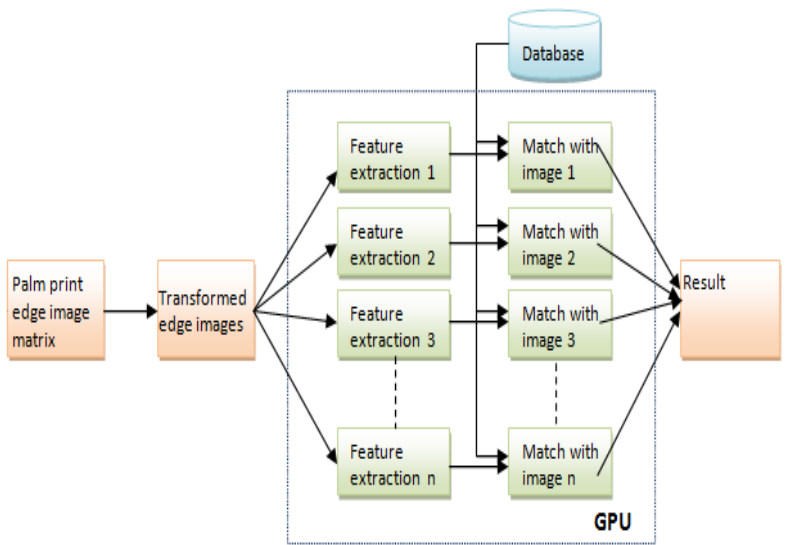

Figure 4. System architecture

Then apply the transform technique on edge image to obtain the edge image transform matrix and finally extract the feature by using the concept of fractional coefficient [1], and store that feature vector in database. While identification, query image feature vector get compared with the feature vector stored in database.

In this proposed system, by using GPU speed to identify the palm image may increase. In this, edge image will be sent to GPU for identification and finally result is sent back from GPU to CPU.

\section{B. $\quad G P U$}

Graphics processing units (GPUs) have emerged as a typical computing resource offering thousands of cores and providing large scale parallelism on computing platform .It is also extremely extensible. The use of GPUs to run all-purpose programs started at premature stage but developers had to consider scientific calculations onto problems that could be represented by vertices and pixels, awaiting NVIDIA launched CUDA [18] in year 2006. 
The only two types of memory that reside on the GPU chip are register and shared memory, global, constant, Local and Texture memory all reside off chip. Local memory is visible only to the thread that wrote it and lasts only for the lifetime of that thread but performs slower. Data which is stored in global memory is visible to all threads within the application and lasts for the duration of the host allocation.

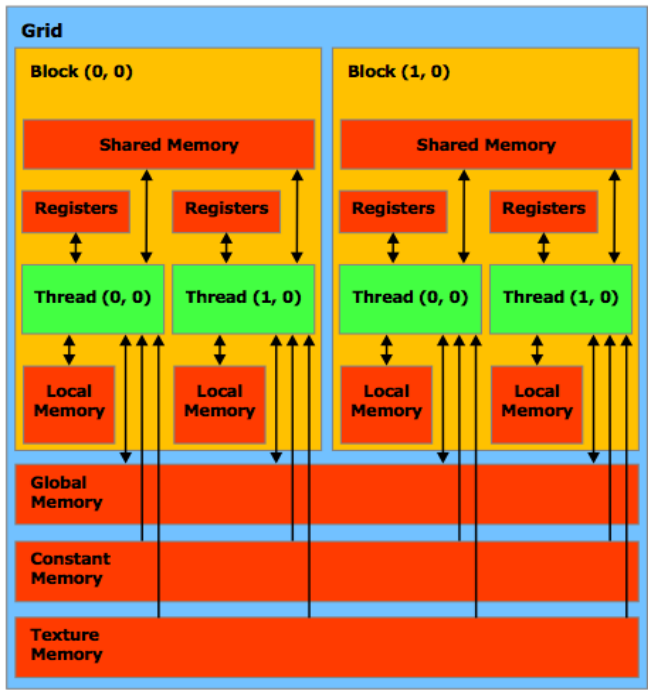

Figure 5. GPU threads and blocks

Single Instruction Multiple Data (SIMD) architecture is used in GPU devices to introduce parallelism. Unlike CPU threads, a context change between two threads is not a costly operation hence simplified computation.

The hardware part of the NVIDIA CUDA represents GPU as an array of streaming multiprocessors and the software side is an extension of the known $\mathrm{C}$ programming.

The GPU implementation for the implementation of code involves the use of the Global memory which works along with the input source and match with the existing images in the database. In an analogous way to the resemblance computation, the first index is to extract the feature vectors of the palm print image and the second to identify the palm print.

\section{CONCLUSION}

Palm print identification has considerable potential as a personal identification technique. It shares most of the discriminative features with fingerprints and in addition possesses a much larger skin area and other discriminative features such as principal lines, ridges and wrinkles. Recent work involves use of transform techniques and multi-resolution based techniques like wavelets have proved to be efficient in implementation of palm print identification and in future use of GPU for parallel computation in feature matching techniques is going to improve the performance and efficiency of the system.

\section{REFERENCES}

[1]. Dr. Sudeep Thapade, Santwana Gudadhe, "Palm Print Identification usingFractional Coefficient of Transformed Edge Palm Images with Cosine,Haar and Kekre Transform", Processidings in IEEE Conference on Information and Communication Technologies , ICT-2013.

[2]. Dr. H. B. Kekre, Dr.Tanuja K. Sarode, Aditya A. Tirodkar, "An Evaluationof Palm Print Recognition Techniques using DCT, Haar Transform andDCT Wavelets and their Performance with Fractional Coefficients",IJCA (0975 - 8887) Volume 32-No.1, October 2011.

[3]. Dr.H.B. Kekre, Dr.Tanuja Sarode, Aditya Tirodkar, "Performance Enhancement of Fractional Coefficients of the Discrete Hartley Transform for Palm Print Recognition", Journal of Telecommunication, Vol.11, Issue1, October2011.

[4]. http://www.digital-systems-lab.net/doc/shk_hartleytransform.pdf

[5]. Dr.Kekre.H ; Thepade.S ; Maloo.A (2005): "Image Retrieval using Fractional Coefficients of Transformed Image using DCT and Walsh Transform", International Journal of Engineering Science and Technology (IJEST), Volume 2, Number 4, 2010, pp.362371.(ISSN:0975-5462).

[6]. Dr Kekre.H; Thepade.S; Sarode.T (2008): "DCT applied to column mean and row mean vectors pof image for fingerprint identification", InternationalConference on computer networks and security, VIT, Pune.

[7]. IIT Delhi Touchless Palmprint Database(Version 1.0), http://web.iitd.ac.in/ ajaykr/Database_Palm.htm

[8]. CASIA palm print data base http://biometrics.idealtest.org/dbDetailForUser.do?id=5

[9]. Liang Wang and Xin Geng, (2010) "Behavioral Biometrics for Human Identification", Medical Information Science Reference,

[10]. Anil K. Jain, Fellow, Arun Ross, Member, Salil Prabhakar, "AnIntroduction to Biometric Recognition", IEEE Transaction on Circuit and System for VideoTechnology, Vol.14, No.1, January 2004.

[11]. X. Wu, K. Wang, D. Zhong "Line feature extraction and matching in palmprint" in: Proceeding of the second international conference on Image \& Graphics 2002 pp. 583-590

[12]. S.Y. Kung, S.H. Lin, M. Fang, A neural network approach to face/palm recognition, in: Proceedings of IEEE Workshop Neural Networks for Signal Processing, (1995), pp. 323-332.

[13]. D.S. Huang, W. Jia, D. Zhang, Palmprint verification based on principal lines, Pattern Recognition 41 (4) (2008) 1316-1328.

[14]. J.Canny. "A computational approach to edge detection", IEEE Transactions on Pattern Analysis and Machine Intelligence, vol.- 8

[15]. W.W. Boles, S.Y.T. Chu, "Personal identification using images of the human palms", in: Proceedings of IEEE Region 10 Annual Conference, Speech and Image Technologies for Computing and Telecommunications, vol. 1, 1997, pp. 295-298.

[16]. D. Zhang, W.K. Kong, J. You, M. Wong on "Line palm print identification",- IEEE Transaction on pattern analysis \& machine Intelligence 25(9) 2003 1041-1051

[17]. A. Kong, D. Zhong, G.Lu (2006) "-A study of identical twins palmprint for personal verification", Pattern recognition 39(11) 2149-2156. NVIDIA Corporation, Santa Clara, CA, USA [Online]. Available: http://www.nvidia.com/

[18]. G. Lu, D, Zhang, K. Wang, "Palm print recognition using Eigen palms feature", Pattern Recognition Letters 24 (9) (2003) 1463-1467

[19]. Adam Kong, David Zhang, and Mohamed Kamel (2006), "Palm print Identification using feature-level fusion",Elsevier Pattern Recognition 39478-487 